\title{
Short-periodic-orbit method for excited chaotic eigenfunctions
}

\author{
F. Revuelta $\odot,{ }^{1, *}$ E. Vergini, ${ }^{2, \dagger}$ R. M. Benito $\odot,{ }^{1, \star}$ and F. Borondo $\oplus^{3,4, \S}$ \\ ${ }^{1}$ Grupo de Sistemas Complejos, Escuela Técnica Superior de Ingeniería Agronómica, Alimentaria y de Biosistemas, \\ Universidad Politécnica de Madrid, Avenida Puerta de Hierro 2-4, 28040 Madrid, Spain \\ ${ }^{2}$ Departamento de Física, Comisión Nacional de Energía Atómica, Avenida del Libertador 8250, 1429 Buenos Aires, Argentina \\ ${ }^{3}$ Instituto de Ciencias Matemáticas (ICMAT), Cantoblanco, 28049 Madrid, Spain \\ ${ }^{4}$ Departamento de Química, Universidad Autónoma de Madrid, Cantoblanco, 28049 Madrid, Spain
}

(Received 17 May 2019; revised 21 August 2020; accepted 18 September 2020; published 9 October 2020)

\begin{abstract}
An alternative method for the calculation of excited chaotic eigenfunctions in arbitrary energy windows is presented. We demonstrate the feasibility of using wave functions localized on unstable periodic orbits as efficient basis sets for this task in classically chaotic systems. The number of required localized wave functions is only of the order of the ratio $t_{H} / t_{E}$, with $t_{H}$ the Heisenberg time and $t_{E}$ the Ehrenfest time. As an illustration, we present convincing results for a coupled two-dimensional quartic oscillator with chaotic dynamics.
\end{abstract}

DOI: 10.1103/PhysRevE.102.042210

\section{INTRODUCTION}

The accurate computation of the eigenstates of a dynamical system is a central problem in physics and computational chemistry. The usual standard methods [1] are based on the variational principle, which implies that in order to get an approximation to the $N$ th eigenstate, the $N-1$ lower-lying ones should also be calculated. This makes the task particularly demanding when one is interested in excited states. The existence of a large density of states or classically chaotic dynamics also significantly contributes to the computational burden. In the case of systems with hard wall boundaries, such as quantum billiards, eigenstate computations are not performed by the variational procedure, so then efficient methods have been developed to calculate only the states within specific energy windows [2]. This fact dramatically reduces the computational time for arbitrarily excited levels, and that fully opened the possibility of studying these interesting states. Unfortunately, such effective methods are not readily available for systems with continuous potentials.

In this paper, we present a method which extends the procedure recently developed by us $[3,4]$ for the computation of eigenstates of classically chaotic systems. This procedure is based on the use of wave functions localized on unstable periodic orbits (POs), which leads to small basis sizes, of the order of the ratio $t_{H} / t_{E}$ between the Heisenberg, $t_{H}$, and the Ehrenfest, $t_{E}$, times. Recall that while the former provides a timescale at which the eigenenergies are accurately obtained, which scales as $t_{H} \sim \mathcal{O}\left(\hbar^{1-d}\right)$, with $d$ the number of degrees of freedom of the system, the latter gives a time lapse where the semiclassical evolution of a wave packet is valid, which

\footnotetext{
*fabio.revuelta@upm.es

†vergini@tandar.cnea.gov.ar

†rosamaria.benito@upm.es

§f.borondo@uam.es
}

is $t_{E} \sim \mathcal{O}(\log \hbar)$. A specific definition of these two important characteristic times for our system will be given below in Sec. II.

The efficiency of the method is demonstrated by a systematic application to small energy windows defined in different energy ranges of a two-dimensional coupled quartic oscillator with highly chaotic dynamics. The key point for the success of our method is the strong energy localization of our basis functions. This interesting property has enabled the calculation of the eigenfunctions of other chaotic systems, such as billiards [2], maps [5], or molecules [4,6], which makes us expect the general validity of our approach. Other attempts to use phase-space basis functions have been made previously in the literature, especially in relation to molecular systems. For example, Davis and Heller used nonorthogonal complex Gaussians, which render analytical expressions for the elements of the Hamiltonian matrix in the case of polynomial potentials [7]. Reimers and Heller extended those semiclassical basis sets for rigid rotors [8]. Wavelets defined in the phase space have been also successfully applied in a series of papers by Poirier and co-workers [9-11]. Similarly, phase-space Gaussians were also applied, on the one hand, by Halverson and Poirier [12], by incorporating some of their ideas previously used with wavelets, and, on the other hand, by Shimshovitz, Bâcić, and Tannor [13,14], using the von Neumann lattice. More recently, Brown and Carrington $[15,16]$ have developed an iterative eigensolver which boosts the numerical calculations. Other long-standing methods for computing molecular energies are the discrete variable representation (DVR) [17,18] using localized basis elements, for which the kinetic and potential energy contributions are easily computed, and the use of the powerful Lanczos diagonalization method [19].

Unstable POs are a cornerstone of classical and quantum chaotic dynamics [20]. They are the only remnants of order in the chaotic sea, and their invariant manifolds organize the classical dynamics in their neighborhoods [21]. From a 
quantum mechanical point of view, POs have also been shown to have a deep impact on the energy levels of classically chaotic systems [20], and also on the characterization of many of their eigenfunctions, where they can yield "scars" on the probability distributions, as shown by Heller in his seminal paper [22]. Bogomolny showed that this localization phenomenon can also arise in a combination of groups of eigenfunctions [23], and later Berry developed the corresponding phase-space version of this theory [24].

Different methods have been reported in the literature for the construction of localized states over unstable POs (hereafter referred to as "scar functions" due to their similarity with Heller's scarring on eigenfunctions). Some use averages over groups of eigenfunctions around the PO quantization condition [25]; others make use of the short PO theory [26,27], apply the asymptotic boundary layer [28], or perform the quantum propagation of wave packets launched along the $\mathrm{PO}$ of interest $[6,29]$.

In this paper, we discuss the spectral properties of our basis functions, and demonstrate the accuracy of the proposed method by carrying out a systematic study of the convergence, both for eigenenergies and eigenfunctions in a small energy window. We also show the feasibility of using remarkably small basis sizes to calculate arbitrarily excited states. Only a brief description of the most relevant computational details and results are given in the text. Let us remark that the method reported would render even more accurate results in the case of more generic systems that present regular and irregular motion at the same time because of the more simple nodal structure of their eigenstates.

The rest of this work is organized as follows. First, we briefly describe in the next section the system under study, which is an anharmonic potential. Second, we describe in Sec. III the method that we have develop to construct efficient basis sets, in the sense that their sizes are small compared to the number of accurately computed eigenfunctions. Third, Sec. IV is devoted to the results and the corresponding discussion. Finally, we sum up the paper in Sec. V with the conclusions and outlook.

\section{SYSTEM}

The system we have chosen to study is described by the classical Hamiltonian

$$
\mathcal{H}\left(p_{x}, p_{y}, x, y\right)=\frac{p_{x}^{2}+p_{y}^{2}}{2}+\frac{x^{2} y^{2}}{2}+\frac{x^{4}+y^{4}}{400} .
$$

The dynamics of this system is extremely chaotic, and no signs of regularity in phase space are observed at first sight [21].

The eigenfunctions of the associated quantum Hamiltonian belong to the five irreducible representations of the $C_{2 v}$ symmetry group. Nevertheless, we only consider here the totally symmetric (even on the axes, $x$ and $y$, and the diagonals, $x=$ $\pm y$ ) eigenfunctions, which belong to the $A_{1}$ representation.

The mean number of $A_{1}$ eigenenergies smaller than $E$ is semiclassically estimated using the method reported in the Ref. [30] as $\mathcal{N}(E) \simeq 0.251 E^{3 / 2} / \hbar^{2}+0.605 E^{3 / 4} / \hbar$. Then, the mean energy density is given by $\rho(E)=d \mathcal{N}(E) / d E \simeq$ $0.376 E^{1 / 2} / \hbar^{2}+0.454 E^{-1 / 4} / \hbar$, while the Heisenberg time is defined by the relationship

$$
t_{H}=2 \pi \hbar \rho(E) .
$$

For the construction of the semiclassical basis one selects a set of unstable short POs in such a way that the sum of their periods is greater than $t_{H}[31]$,

$$
\sum_{\gamma} T_{\gamma}>t_{H}
$$

where $T_{\gamma}$ is the period of a short $\mathrm{PO} \gamma$. At this point it is worth noticing that the monodromy method $[32,33]$ is exceptionally fast for calculating POs in a general Hamiltonian system, especially in high dimensions. Nevertheless, here the situation is much more simple because our Hamiltonian has only two degrees of freedom and it presents the symmetries of the $C_{4 v}$ point group. As a consequence, the shortest POs, which are symmetrical, are trivially evaluated. Furthermore, the Hamiltonian (1) is mechanically similar, and then it presents no bifurcations as a function of the energy. Thus, the phase-space structure does not change with the energy, and then one can compute all the POs at a reference energy $E$ and scale them for a different energy $E^{\prime}$ as

$$
\begin{aligned}
& x_{t^{\prime}}^{\prime}=\left(\frac{E^{\prime}}{E}\right)^{1 / 4} x_{t}, \quad p_{x^{\prime}, t^{\prime}}^{\prime}=\left(\frac{E^{\prime}}{E}\right)^{1 / 2} p_{x, t}, \\
& y_{t^{\prime}}^{\prime}=\left(\frac{E^{\prime}}{E}\right)^{1 / 4} y_{t}, \quad p_{y^{\prime}, t^{\prime}}^{\prime}=\left(\frac{E^{\prime}}{E}\right)^{1 / 2} p_{y, t},
\end{aligned}
$$

with $t^{\prime}=\left(E^{\prime} / E\right)^{-1 / 4} t$. Of course the first two periodic orbits of Fig. 1 are given without any calculation, and consequently let us consider, for instance, orbits 3,6 , and 7. By fixing the starting point with $y=0$ and $p_{x}=0$ one looks for the coordinate $x$ in such a way that the first intersection of the trajectory with the diagonal occurs orthogonal to it.

In this paper, we use the POs displayed in Fig. 1, with $\sum T_{\gamma} \simeq 32$ and $t_{H} \simeq 26$ at $E=110$, the maximum energy considered in the calculation. These POs are arranged by the number of bounces with the symmetry lines. Obviously, a criterion of the general validity for arranging short POs with the purpose of covering phase space in an efficient way is desirable. We believe that such a criterion could be defined in terms of the properties of the first homoclinic orbit of each short PO, particularly in a higher dimension. Still, condition (3) and the fact that one only needs the shortest POs of the system strongly limits the time required to search for POs. Moreover, symmetry considerations can further simplify this task.

Associated with an unstable short PO $\gamma$, one obtains the discrete set of Bohr-Sommerfeld (BS) energies $E_{n}^{\gamma}$ computed from the relationship

$$
\frac{S_{\gamma}}{\hbar}-\frac{\mu_{\gamma} \pi}{2}=2 \pi n
$$

with $S_{\gamma}$ the action of $\gamma$ and $\mu_{\gamma}$ its Maslov index.

We provide a semiclassical construction of wave functions with $n$ excitations along $\gamma$, the so-called tube functions of $\gamma$, with mean energy $E_{n}^{\gamma}$ and small energy dispersion given by $\hbar \lambda_{\gamma} / \sqrt{2}$, where $\lambda_{\gamma}$ is the stability exponent of $\gamma$. Although the time evolution of these wave functions can be 


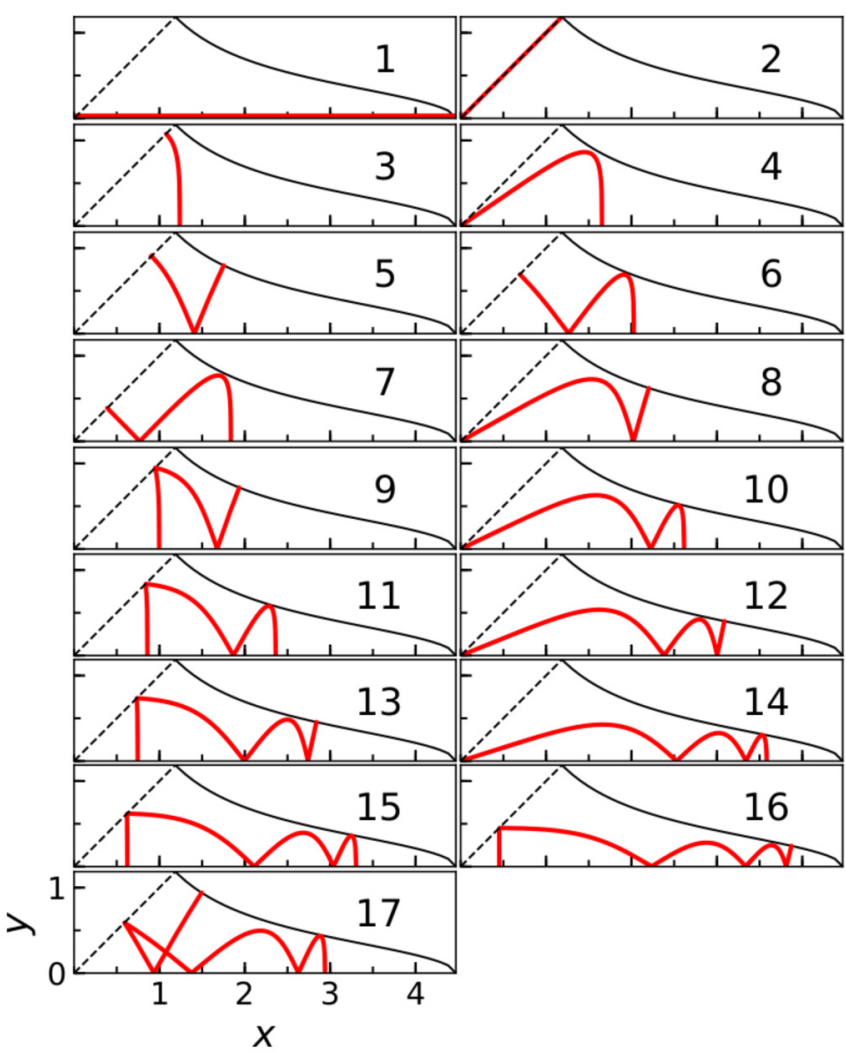

FIG. 1. Set of desymmetrized periodic orbits used in the calculation (red curves). The equipotential at energy $E=1$ for Eq. (1) is shown as a solid black curve.

evaluated at a semiclassical level in terms of homoclinic and heteroclinic orbits $[34,35]$, here we prefer to use a quantum evolution, following Ref. [36], in order to obtain highly accurate eigenfunctions and eigenvalues. In this respect, it is worth mentioning that the quantum evolution of tube functions up to times of the order of the mean Ehrenfest time $t_{E}$, that in our case is given by

$$
t_{E}=\frac{1}{2 \lambda} \ln \left(\frac{\mathcal{A}}{\hbar}\right)
$$

with $\lambda \simeq 0.385 E^{1 / 4}$ the Lyapunov exponent of the system, and $\mathcal{A} \simeq 11.1 E^{3 / 4}$ the area of a characteristic Poincaré surface of the section, is a simple task.

\section{METHOD}

For the computation of eigenvalues and eigenfunctions contained in an energy window defined by the central energy $E_{0}$ and width $\Delta E,\left[E_{0}-\Delta E, E_{0}+\Delta E\right]$, we proceed as follows.

First, we select a set of BS energies of the POs existing in an enlarged energy window $\left[E_{0}-(\Delta E+\delta), E_{0}+(\Delta E+\right.$ $\delta)$ ], with $\delta \simeq 2\left(t_{H} / t_{E}\right) / \rho(E)$ an energy window containing $2 t_{H} / t_{E}$ eigenvalues.

Second, we construct tube functions of the selected BS energies, for instance, $E_{n}^{\gamma}$. By propagating a Gaussian wave packet over $\gamma$ using the frozen Gaussian approximation [37], one has

$$
\psi_{n}^{\gamma}(x, y)=\int_{0}^{T_{\gamma}} e^{i E_{n}^{\gamma} t / \hbar} \phi(x, y, t) d t
$$

where

$$
\begin{aligned}
\phi(x, y, t)= & \exp \left\{-\alpha\left[\left(x-x_{t}\right)^{2}+\left(y-y_{t}\right)^{2}\right]\right. \\
& \left.+i\left[p_{x_{t}}\left(x-x_{t}\right) / \hbar+p_{y_{t}}\left(y-y_{t}\right) / \hbar+2 \pi n t / T_{\gamma}\right]\right\},
\end{aligned}
$$

with $\left(x_{t}, y_{t}, p_{x_{t}}, p_{y_{t}}\right)$ the classical evolution over $\gamma$. The energy dispersion of $\psi_{n}^{\gamma}(x, y)$ behaves as $C \hbar$ in leading order, with $C$ a constant that depends on the parameter $\alpha$ of Eq. (8). By selecting the parameter $\alpha$ that minimizes the energy dispersion for a finite value of $\hbar$, we obtain a good estimate of the parameter that minimizes the coefficient $C$. Of course, the main idea behind this choice is that as the energy dispersion is smaller, the wave function is closer to an eigenfunction. In conclusion, one selects the parameter $\alpha$ in such a way that the energy dispersion of $\psi_{n}^{\gamma}(x, y)$ results in a minimum.

Third, scar functions are constructed by quantum propagation of the tube functions (7) up to $t_{E}$,

$$
\langle x, y \mid \gamma, n\rangle=\int_{-t_{E}}^{t_{E}} \cos \left(\frac{\pi t}{2 t_{E}}\right) e^{i\left(E_{n}^{\gamma}-\hat{\mathcal{H}}\right) t / \hbar} \psi_{n}^{\gamma}(x, y) d t .
$$

This time evolution is the most demanding point of our method, but still it can be efficiently carried out using procedures such as the discrete variable representation $[17,18]$ because of the small energy dispersion of the tube functions [36]. Similar results are expected when other basis functions with similar spectral properties are used, e.g., the scar functions constructed using the method reported in Ref. [26]. Similarly, Eq. (7) could be also used to construct the semiclassical basis set, but the (much) smaller spectral dispersion of the scar functions (9) after a relative short time propagation makes these last functions better suited. As a particular example, notice in Fig. 2(a) that the spectra of four of the scar functions involved in our calculations are mostly concentrated in the window of interest. Here, the infinite resolution spectra are shown as vertical thin lines, while their finite-resolution counterpart, which is defined as the Lorenzian convolution

$$
I_{\Delta}(E)=\frac{\Delta E}{2 \pi} \sum_{N} \frac{\left\langle\psi_{n}^{\gamma} \mid N\right\rangle^{2}}{\left(E-E_{N}\right)^{2}+(\Delta E / 2)^{2}},
$$

with $\Delta E=0.4$ and $E_{N}$ is the energy of the eigenstate $|N\rangle$, are shown as vertical black thick lines at the bottom of Fig. 2(a). Notice that these spectra can only be obtained once the eigenfunctions of the system have been calculated.

Fourth, the Gram-Schmidt selective method [3] is then used to choose the most fit scar functions. The method works as follows. One selects the scar function with minimum energy dispersion as the first function of the basis, for instance, $|\gamma, n\rangle$. Next, one eliminates, for the other scar functions, the contribution along $|\gamma, n\rangle$, for instance, $|\eta, m\rangle$ (with $\eta$ other PO) is replaced by $|\eta, m\rangle-\langle\gamma, n \mid \eta, m\rangle|\gamma, n\rangle$. Then, one evaluates the energy dispersion of the new wave functions and selects as the second function of the basis the one with minimum dispersion. This process continues in a similar way to the Gram-Schmidt method while the minimum energy dispersion 

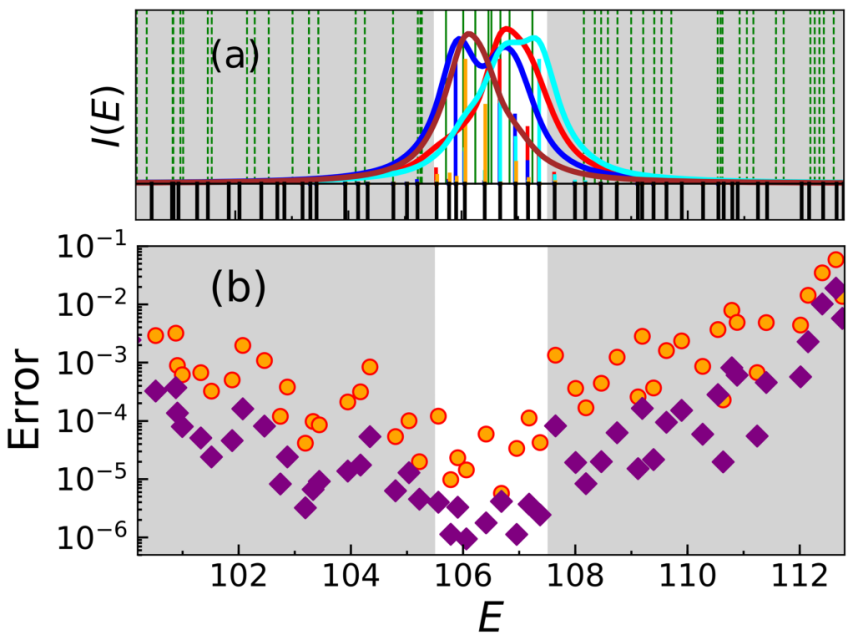

FIG. 2. (a) Bohr-Sommerfeld quantized energies (vertical green lines) for the 61 scar functions in the basis set used to obtain the nine eigenstates of $A_{1}$ symmetry (vertical black lines) of the quartic oscillator (1) lying in the energy window [105.5, 107.5] (between gray shaded regions). The infinite (vertical colored lines) and low-resolution (continuous thick curves) projections on the eigenenergies spectrum of the $|11,44\rangle$ (red), $|5,24\rangle$ (blue), $|14,55\rangle$ (cyan), and $|17,67\rangle$ (brown) scar functions shown in Fig. 4 (see text for notation), which are the ones which are mainly involved in the reconstruction of the eigenfunction 297, have been superimposed. (b) Errors in the computed eigenenergies (orange circles) and eigenfunctions (purple diamonds) given by Eq. (11).

is smaller than a given value, for instance, $2 \delta$. The method has the effect of describing the subspace spanned by the desired eigenfunctions, i.e., those whose energies lie within the energy window under study.

Fifth, once the final basis set is obtained, the corresponding Hamiltonian matrix is computed and diagonalized using standard procedures.

\section{RESULTS AND DISCUSSION}

Figure 3 presents the results of a computation performed in the energy window [105.5,107.5] for $\hbar=1$. There are 66 scar functions contained in the enlarged energy window [100.2, 112.8] [see the vertical green lines in Fig. 2(a)], and the Gram-Schmidt selective method chooses 61 scar functions in order to reproduce with high accuracy the nine eigenvalues [black lines contained in the central white region of Fig. 2(a)] and eigenfunctions of interest. The figure displays the probability density of these eigenfunctions in the fundamental domain. As can be seen, they all present a very complex nodal pattern, which is characteristic of classically chaotic systems. With respect to the scar phenomenon, we clearly observe that the eigenfunction 293 is localized along the first PO of Fig. 1.

The eigenfunction $|j\rangle$ of our computation is compared with the "exact" result $\left|j^{\prime}\right\rangle$ obtained using a basis set of 5000 harmonic oscillator functions. We compute the error of eigenvalues and eigenfunctions by the dimensionless quantities

$$
\rho(E)\left|E_{j}-E_{j^{\prime}}\right| \text { and } 1-\left\langle j \mid j^{\prime}\right\rangle^{2}
$$
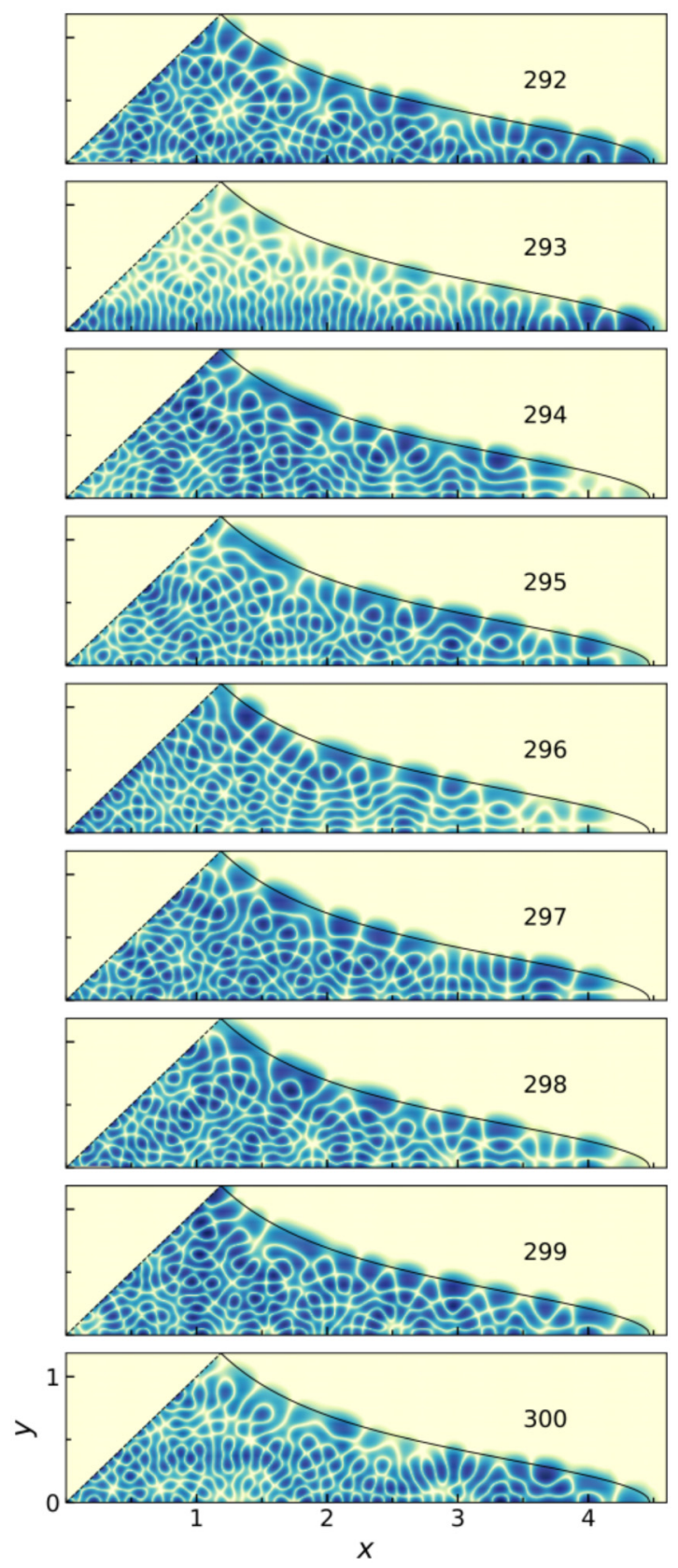

FIG. 3. Probability density of the eigenfunctions contained in the energy window $E=[105.5,107.5]$. They are scaled to $E=1$ for comparison. The corresponding eigenstate number is given in the plots.

respectively. The mean error of the nine eigenvalues and eigenfunctions of Fig. 3 is of order $\sim 10^{-4}$ and $\sim 10^{-5}$, respectively, as shown in Fig. 2(b). A similar accuracy based on the variational principle would require much larger full matrix diagonalizations. In particular, for the energy window reported in this paper, the same level of accuracy requires a harmonic oscillator basis set formed by $\sim 50$ times more basis elements than the one reported here, which exerts a diagonalization time $\sim 50^{3}$ times larger. Furthermore, by accepting an error ten times greater than the previous ones, the same calculation provides 20 consecutive eigenfunctions. With respect to the 

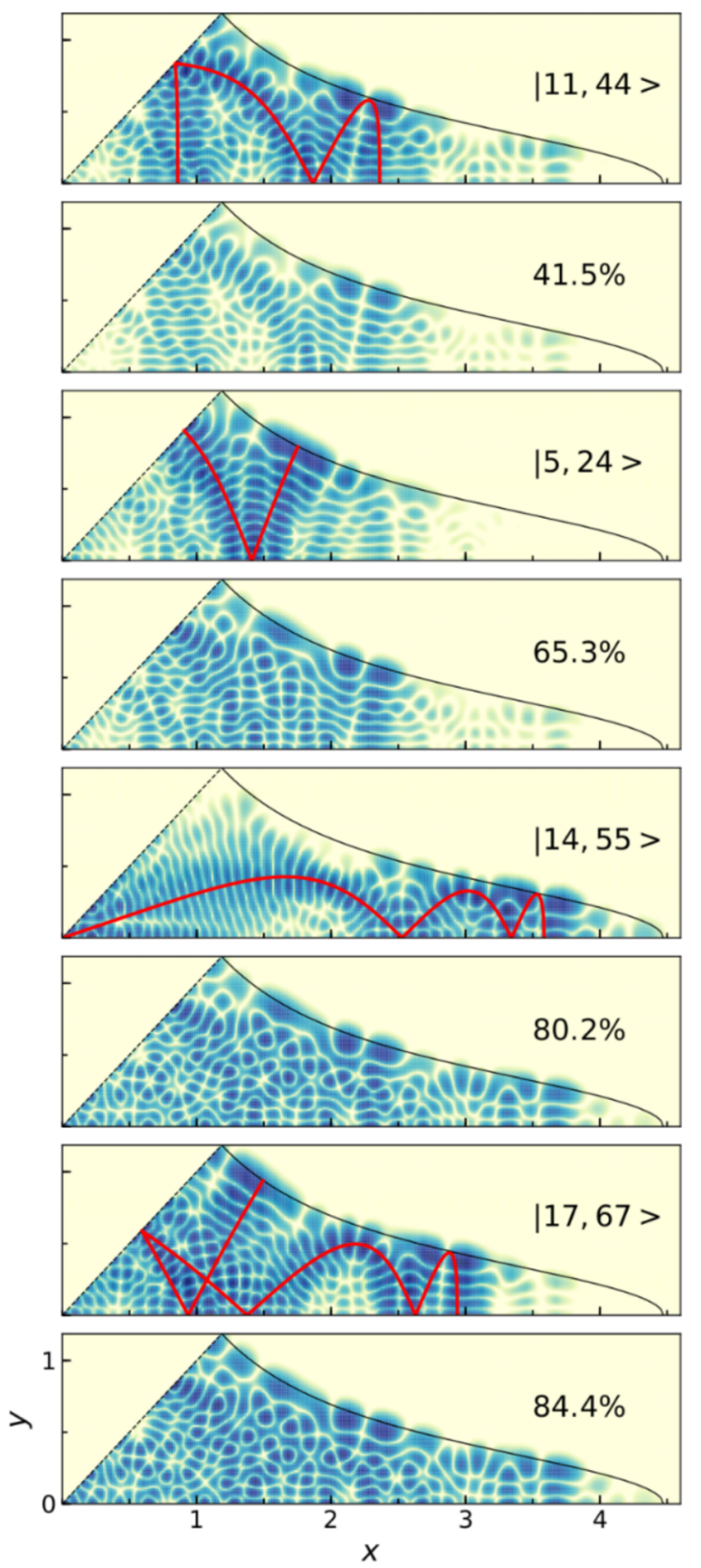

FIG. 4. Partial reconstruction of eigenfunction 297 (see Fig. 3) by incorporating the most relevant scar functions. The percentage of reconstruction at each step is also given.

stability of the method, let us remark that in other energy windows of the spectrum up to $E=110$, we found essentially the same result. Moreover, for smaller energies a reduced number of POs is required as long as Eq. (3) is accomplished. Finally, we find that the dimension of the basis is only $40 \%$ greater than the number of eigenfunctions contained in the enlarged energy window.

Let us consider now the representation of the eigenfunctions obtained in our calculation in the basis set of scar functions, which shows the basis elements that contribute the most (largest overlaps) to their reconstruction. Notice that this procedure provides valuable information on the influence that each PO has on the emergence of the properties of individual

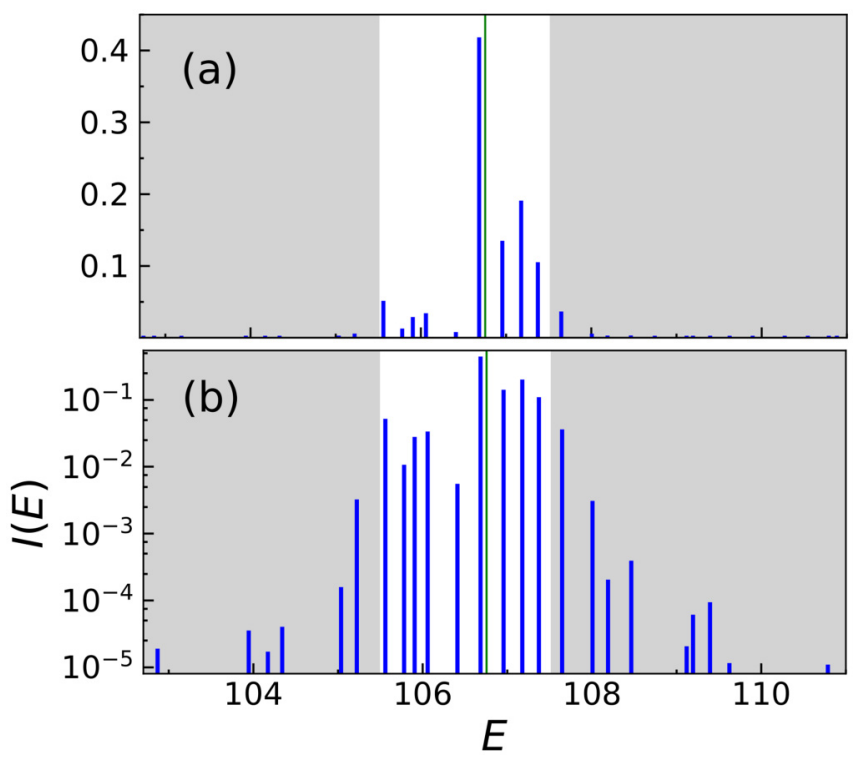

FIG. 5. Square modulus of the overlap of the first wave function of Fig. 4, i.e., the scar function $|11,44\rangle$, with the eigenfunctions of the system, as a function of the eigenenergies in (a) linear and (b) logarithmic scales. The thin green line marks the position of the Bohr-Sommerfeld quantization energy ( $E \simeq 106.75$ ), which lies within the reference energy window $[105.5,107.5]$ (between the gray shaded regions).

chaotic eigenfunctions, which represents an important added bonus of our method to the theory of quantum chaos. As an illustrative example, we present in Fig. 4 the reconstruction of the eigenfunction 297 shown in Fig. 3. As already mentioned, it exhibits a complex nodal pattern, as it is not localized over any single PO. Successive steps in the reconstruction are given in the figure. In each one of them we show the result of adding the scar function depicted in the panel above it. Notice that $41.5 \%$ of the eigenfunction is reconstructed solely with the scar function $|11,44\rangle$. By adding the scar function $|5,24\rangle$, $65.3 \%$ of the eigenfunction is reconstructed. This figure increases when more basis functions are included, up to a value of $84.4 \%$ when four basis functions are combined. In other words, the square modulus of the projection of the eigenstate 297 onto the subspace spanned by the four scar functions of the figure is 0.844 . Notice also how the reconstruction procedure that we have just described progressively refines the intricate details of the eigenfunction.

The other eigenfunctions of Fig. 3 have a similar partial reconstruction in terms of a small number of scar functions. In particular, we remark on the cases where only one scar function is able to reproduce more than $50 \%$ of the eigenfunction. The scar function $|1,56\rangle$ reproduces $79.7 \%$ of 293 , $|8,41\rangle$ reproduces $60.6 \%$ of 296 , and $|16,56\rangle$ reproduces $58.9 \%$ of 300 .

In order to clearly show the strong energy localization of our basis functions, we conclude this section by displaying in Fig. 5 the square modulus of the overlap of the first wave function in Fig. 4, i.e., the scar function $|11,44\rangle$, with the eigenfunctions of the system. In Fig. 5(a) we use a linear scale to remark on the fact that the energy dispersion of the wave function consists only of contributions within a few 
mean-level spacings. Notice here that the highest peak lies very close to the Bohr-Sommerfeld energy of the scar function (thin green line at $E \simeq 106.75$ ), and corresponds to the overlap associated with the eigenstate 297 . Recall that the scar function $|11,44\rangle$ is precisely the main contribution to the reconstruction of that eigenstate. Furthermore, in Fig. 5(b) we use a logarithmic scale to emphasize that the intensities decay exponentially as we move away from the corresponding Bohr-Sommerfeld energy.

\section{CONCLUSIONS AND OUTLOOK}

The short-periodic-orbit method, formulated and tested in this paper, is an extremely powerful technique for calculating very excited chaotic eigenfunctions. We have shown the feasibility of computing eigenfunctions of a classically chaotic system using small basis sets. The key point is the use of scar functions constructed in the neighborhood of a set of short periodic orbits that covers the relevant phase space efficiently. These semiclassical functions have excellent properties regarding localization in energy that make them suitable to describe only the portion of the system's Hilbert space spanned by the eigenstates of interest. In particular, the accuracy of the method has been demonstrated by performing a systematic calculation of the eigenfunctions of a coupled quartic oscillator using narrow energy windows in excited regions of the spectrum. Let us finally remark that each eigenfunction obtained in the calculation is partially reconstructed using only a few scar functions, of the order of the $t_{H} / t_{E}$ ratio. This fact provides a useful way of classifying chaotic eigenfunctions.

\section{ACKNOWLEDGMENTS}

This work has been partially supported by the Ministerio de Economía, Competitividad e Innovación (MINECO) of the Gobierno de España under Contract No. MTM2015-63914-P, by the Ministerio de Ciencia, Innovación y Universidades under Contract No. PGC2018-093854-B-I00, and by ICMAT Severo Ochoa under Contracts No. SEV-2015-0554 and No. CEX2019-000904-S. F.R. also acknowledges the financial support of the programs to improve the research of young doctors of the Programa Propio of the Universidad Politécnica de Madrid, and of the project GeoCoSiM, financed under the Plurianual Agreement between the Comunidad de Madrid and the Universidad Politécnica de Madrid.
[1] Y. Shao et al., Advances in methods and algorithms in a modern quantum chemistry program package, Phys. Chem. Chem. Phys. 8, 3172 (2006).

[2] E. Vergini and M. Saraceno, Calculation by scaling of highly excited states of billiards, Phys. Rev. E 52, 2204 (1995).

[3] F. Revuelta, R. M. Benito, F. Borondo, and E. Vergini, Using basis sets of scar functions, Phys. Rev. E 87, 042921 (2013).

[4] F. Revuelta, E. Vergini, R. M. Benito, and F. Borondo, Semiclassical basis sets for the computation of molecular vibrational states, J. Chem. Phys. 146, 014107 (2017).

[5] L. Ermann and M. Saraceno, Periodic orbit basis for the quantum Baker map, Phys. Rev. E 78, 036221 (2008).

[6] F. Revuelta, E. Vergini, R. M. Benito, and F. Borondo, Scar functions, barriers for chemical reactivity, and vibrational basis sets, J. Phys. Chem. A 120, 4928 (2016).

[7] M. J. Davis and E. J. Heller, Semiclassical Gaussian basis set method for molecular vibrational wave functions, J. Chem. Phys. 71, 3383 (1979).

[8] J. R. Reimers and E. J. Heller, The exact eigenfunctions and eigenvalues of a two-dimensional rigid rotor obtained using Gaussian wave packet dynamics, J. Chem. Phys. 83, 511 (1985).

[9] B. Poirier and A. Salam, Quantum dynamics calculations using symmetrized, orthogonal Weyl-Heisenberg wavelets with a phase space truncation scheme. II. Construction and optimization, J. Chem. Phys. 121, 1690 (2004).

[10] B. Poirier and A. Salam, Quantum dynamics calculations using symmetrized, orthogonal Weyl-Heisenberg wavelets with a phase space truncation scheme. III. Representations and calculations, J. Chem. Phys. 121, 1704 (2004).

[11] T. Halverson and B. Poirier, Calculation of exact vibrational spectra for $\mathrm{P}_{2} \mathrm{O}$ and $\mathrm{CH}_{2} \mathrm{NH}$ using a phase space wavelet basis, J. Chem. Phys. 140, 204112 (2014).

[12] T. Halverson and B. Poirier, Accurate quantum dynamics calculations using symmetrized Gaussians on a doubly dense von Neumann lattice, J. Chem. Phys. 137, 224101 (2012).

[13] A. Shimshovitz and D. J. Tannor, Phase-Space Approach to Solving the Time-Independent Schrödinger Equation, Phys. Rev. Lett. 109, 070402 (2012).

[14] A. Shimshovitz, Z. Bâcić, and D. J. Tannor, The von Neumann basis in non-Cartesian coordinates: Application to floppy triatomic molecules, J. Chem. Phys. 141, 234106 (2014).

[15] J. Brown and T. Carrington, Using an iterative eigensolver to compute vibrational energies with phase-spaced localized basis functions, J. Chem. Phys. 143, 044104 (2015).

[16] J. Brown and T. Carrington, Assessing the utility of phasespace-localized basis functions: Exploiting direct product structure and a new basis function selection procedure, J. Chem. Phys. 144, 244115 (2016).

[17] R. G. Littlejohn, M. Cargo, T. Carrington, K. A. Mitchell, and B. Poirier, A general framework for discrete variable representation basis sets, J. Chem. Phys. 116, 8691 (2002).

[18] Z. Bâcić and J. C. Light, Theoretical methods for rovibrational states of floppy molecules, Annu. Rev. Phys. Chem. 40, 469 (1989).

[19] X.-G. Wang and T. Carrington, A contracted basis-Lanczos calculation of vibrational levels of methane: Solving the Schrödinger equation in nine dimensions, J. Chem. Phys. 119, 101 (2003).

[20] M. C. Gutzwiller, Chaos in Classical and Quantum Mechanics (Springer, New York, 1990).

[21] A. J. Lichtenberg and M. A. Lieberman, Regular and Stochastic Motion (Springer, New York, 2010).

[22] E. J. Heller, Bound-State Eigenfunctions of Classically Chaotic Hamiltonian Systems: Scars of Periodic Orbits, Phys. Rev. Lett. 53, 1515 (1984).

[23] E. B. Bogomolny, Smoothed wave functions of chaotic quantum systems, Physica D 31, 169 (1988). 
[24] M. V. Berry, Quantum scars of classical closed orbits in phase space, Proc. R. Soc. London, Ser. A 423, 219 (1989).

[25] G. G. de Polavieja, F. Borondo, and R. M. Benito, Scars in Groups of Eigenstates in a Classically Chaotic System, Phys. Rev. Lett. 73, 1613 (1994).

[26] E. G. Vergini and G. G. Carlo, Semiclassical construction of resonances with hyperbolic structure: The scar function, J. Phys. A: Math. Gen. 34, 4525 (2001).

[27] G. G. Carlo, E. G. Vergini, and P. Lustemberg, Scar functions in the Bunimovich stadium billiard, J. Phys. A: Math. Gen. 35, 7965 (2002).

[28] A. Vagov, H. Schomerus, and V. V. Zalipaev, Asymptoticboundary-layer method for unstable trajectories: Semiclassical expansions for individual scar wave functions, Phys. Rev. E 80, 056202 (2009).

[29] F. Revuelta, E. G. Vergini, R. M. Benito, and F. Borondo, Computationally efficient method to construct scar functions, Phys. Rev. E 85, 026214 (2012).

[30] O. Bohigas, S. Tomsovic, and D. Ullmo, Manifestations of classical phase space structures in quantum mechanics, Phys. Rep. 223, 43 (1993).
[31] E. G. Vergini, Semiclassical theory of short periodic orbits in quantum chaos, J. Phys. A: Math. Gen. 33, 4709 (2000).

[32] M. Baranger, K. T. R. Davies, and J. H. Mahoney, The calculation of periodic trajectories, Ann. Phys. (NY) 186, 95 (1988).

[33] K. T. R. Davies, T. E. Huston, and M. Baranger, Calculations of periodic trajectories for the Hénon-Heiles Hamiltonian using the monodromy method, Chaos 2, 215 (1992).

[34] E. G. Vergini, Semiclassical Approach to Long Time Propagation in Quantum Chaos: Predicting Scars, Phys. Rev. Lett. 108, 264101 (2012).

[35] E. G. Vergini, Semiclassical propagator to evaluate off-diagonal matrix elements of the evolution operator between quantum states, Phys. Rev. E 89, 020901 (2014).

[36] E. G. Vergini, E. L. Sibert III, F. Revuelta, R. M. Benito, and F. Borondo, Diagonal matrix elements in a scar function basis set, Europhys. Lett. 89, 40013 (2010).

[37] E. J. Heller, Time-dependent approach to semiclassical dynamics, J. Chem. Phys. 62, 1544 (1975). 